\title{
Brain White Matter Lesions Classification in Multiple Sclerosis Subjects for the Prognosis of Future Disability
}

\author{
Christos P. Loizou ${ }^{1}$, Efthyvoulos C. Kyriacou ${ }^{2}$, Ioannis Seimenis ${ }^{3}$, Marios Pantziaris ${ }^{4}$, \\ Christodoulos Christodoulou ${ }^{5}$, and Constantinos S. Pattichis ${ }^{5}$ \\ ${ }^{1}$ Department of Computer Science, Intercollege, P.O. Box 51604, \\ CY-3507, Limassol, Cyprus \\ panloicy@logosnet.cy.net \\ ${ }^{2}$ Department of Computer Science and Engineering, \\ Frederick University, Limassol, Cyprus \\ ${ }^{3}$ Medical Diagnostic Centre "Ayios Therissos", \\ 2033 Nicosia, Cyprus \\ ${ }^{4}$ Cyprus Institute of Neurology and Genetics, Nicosia, Cyprus \\ ${ }^{5}$ Department of Computer Science, University of Cyprus, Nicosia, Cyprus
}

\begin{abstract}
This study investigates the application of classification methods for the prognosis of future disability on MRI-detectable brain white matter lesions in subjects diagnosed with clinical isolated syndrome (CIS) of multiple sclerosis (MS). For this purpose, MS lesions and normal appearing white matter (NAWM) from 30 symptomatic untreated MS subjects, as well as normal white matter (NWM) from 20 healthy volunteers, were manually segmented, by an experienced MS neurologist, on transverse T2-weighted images obtained from serial brain MR imaging scans. A support vector machines classifier (SVM) based on texture features was developed to classify MRI lesions detected at the onset of the disease into two classes, those belonging to patients with EDSS $\leq 2$ and EDSS $>2$ (expanded disability status scale (EDSS) that was measured at 24 months after the onset of the disease). The highest percentage of correct classification's score achieved was $77 \%$. The findings of this study provide evidence that texture features of MRI-detectable brain white matter lesions may have an additional potential role in the clinical evaluation of MRI images in MS. However, a larger scale study is needed to establish the application of texture analysis in clinical practice.
\end{abstract}

Keywords: MRI, multiple sclerosis, texture classification.

\section{Introduction}

Multiple sclerosis (MS) is the most common autoimmune disease of the central nervous system, with complex pathophysiology, including inflammation, demyelination, axonal degeneration, and neuronal loss. Within individuals, the clinical manifestations are unpredictable, ${ }^{1}$ particularly with regard to the development of disability [1]. 
Diagnostic evaluation of MS, performed by a specialized neurologist, is generally based on conventional magnetic resonance imaging (MRI) following the McDonald criteria [2], and on clinical signs and symptoms. The development of modern imaging techniques for the early detection of brain inflammation and the characterization of tissue-specific injury is an important objective in MS research. Recent MRI studies have shown that brain and focal lesion volume measures, magnetization transfer ratio and diffusion weighted imaging-derived parameters can provide new information in diagnosing MS [3]. Texture features quantify macroscopic lesions and also the microscopic abnormalities that may be undetectable using conventional measures of lesion volume and number [1], [4]. Several studies have been published, where it was documented that texture features can be used for the assessment of MS lesions in: (i) differentiating between lesions for normal white matter (NWM), and the so called normal appearing white matter (NAWM) [5-9], and (ii) monitoring the progression of the disease over longitudinal scans [10-14].

Our primary objective in this study is to develop texture classification methods that can be used to predict MS brain lesions that at a later stage are associated with advanced clinical disability and more specifically with the extended disability status scale (EDSS). Since the use of quantitative MRI analysis as a surrogate outcome is used as a surrogate measure in clinical trials, we hypothesise that there is a close relationship between the change in the extracted features and the clinical status and the rate of development of disability. We analyzed patient's images acquired at the initial stages of the disease-clinical isolated syndrome (CIS) (0 months) and we correlated texture findings with disability assessment scales. We interrelate therefore the EDSS scores [15] with standard shape and texture features.

Preliminary findings of this study, for the texture analysis of NAWM in MS subjects were also published in [8], [9]. It should be note that the same problem was also investigated in a recent study published by our group using Amplitude Modulation-Frequency Modulation (AM-FM) analysis [14]. The motivation of this study was to investigate the usefulness of classical texture analysis, as well as compare the findings with the AM-M analysis.

MRI-based texture analysis was shown to be effective in classifying MS lesions from NWM and the so called, NAWM, with an accuracy of 96\%-100\% 5. In [6], the authors showed that texture features can reveal discriminant features for differentiating between normal and abnormal tissue, and for image segmentation. Significant differences in texture between normal and diseased spinal cord in MS subjects were found in [7] as well a significant correlation between texture features and disability. The median value increase of these texture features suggests that the lesions texture in MS subjects is less homogeneous and more complex than the corresponding healthy tissue (NWM) in healthy subjects [7]. Similar findings were also found in [8] and [9], where it was shown that median values of lesion texture features such as standard deviation, median, sum of squares variance, contrast, sum average, sum variance, difference variance, and difference entropy increased significantly with the progression of the MS disease when compared to NWM tissue. Statistical analysis (using the spatial gray level dependence matrices) has shown that there is a significant difference between lesions and NAWM or NWM. These findings may be beneficial in the research of early diagnosis and treatment monitoring in MS. 
The differentiation between active and non-active brain lesions in MS subjects from brain MRI was also investigated in [11]. Here, it was shown that active lesions could be identified without frequent gadolinium injections, using run length analysis criteria. In [12] the performance of texture analysis concerning discrimination between MS lesions, NAWM and NWM from healthy controls was investigated by using linear discriminant analysis. The results suggested that texture features can support early diagnosis in MS. When a combined set of texture features was used [12], similar findings were reported.

In [13], a pattern recognition system was developed for the discrimination of multiple sclerosis from cerebral microangiopathy lesions based on computer-assisted texture analysis of magnetic resonance images. It was shown that, MS regions were darker, of higher contrast, less homogeneous and rougher as compared to cerebral microangiopathy. Finally, in [14], the use of multi-scale Amplitude ModulationFrequency Modulation (AM-FM) texture analysis of MS using magnetic resonance images from brain was introduced.

\section{Materials and Methods}

\subsection{Material and MRI Acquisition}

Thirty subjects (15 males, and 15 females), aged 31.4 \pm 12.6 (mean age \pm standard deviation) were scanned at $1.5 \mathrm{~T}$ within one month following initial clinical evaluation to confirm CIS diagnosis. The transverse MR images used for analysis were obtained using a T2-weighted turbo spin echo pulse sequence (repetition time $=4408 \mathrm{~ms}$, echo time $=100 \mathrm{~ms}$, echo spacing=10.8 ms). The reconstructed image had a slice thickness of $5 \mathrm{~mm}$ and a field of view of $230 \mathrm{~mm}$ with a pixel resolution of 2.226 pixels per $\mathrm{mm}$. Standardized planning procedures were followed during each MRI examination. The MRI protocol and the acquisition parameters were given in detail in [8], [9].

Initial clinical evaluation was made by an experienced MS neurologist (co author M. P.) who referred subjects for a baseline MRI upon diagnosis and clinically followed all subjects for over two years. All subjects were subjected to an EDSS test two years after initial diagnosis to quantify disability [15].

A normalization algorithm was used to match the image brightness between the first (baseline) and the follow-up images (see [16] for details). For this purpose, the neurologist manually segmented cerebrovascular fluid (CSF) areas as well as areas with air (sinuses) from all MS brain scans. Similarly, ROIs representing NWM, CSF and air from the sinuses were arbitrarily segmented from the brain scans of the 20 healthy subjects. The original image histogram was stretched, and shifted in order to cover all the gray scale levels in the image.

To introduce the objective of our study, an example in Fig. 1 is presented. Here, we show a transaxial T2-weighted MR image of a female patient at the age of 32, with an EDSS [15] equal to 3. The image in Fig. 1a) corresponds to the initial diagnosis of a CIS of MS and the delineated lesion corresponding to the MS plaque is also depicted. Figure 1b) shows the magnified segmented lesion from a) (original images were acquired at a sampling rate of 2.226 pixels per $\mathrm{mm}$ ). In what follows, texture analysis refers to the image processing of the extracted regions of interest (ROIs). 


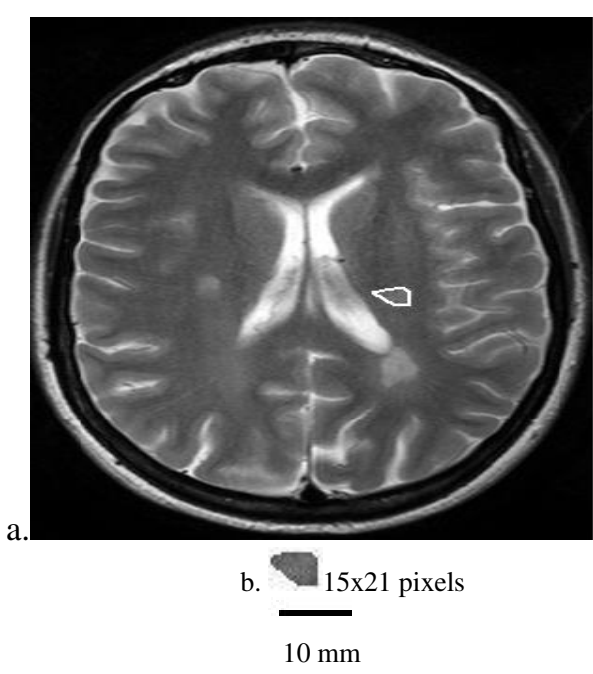

Fig. 1. a) ROI drawn on MR image of the brain obtained from a 32 year old female MS patient with an EDSS $=3$ at 0 months, and b) magnified segmented lesion that was acquired at a pixel resolution of 2.226 pixels per $\mathrm{mm}$. The bar below the lesion shows the size of 10 $\mathrm{mm}$. The grey scale median and inter-quartile range (IQR) of the segmented lesion were 108 and 9.6, respectively

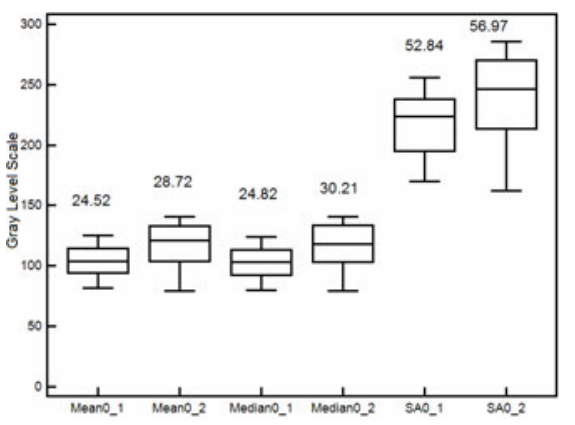

Fig. 2. Box plots for the median \pm interquartile range (IQR) values for texture features mean, median and sum average, from MS lesions at 0 months for EDSS $<=2(\mathrm{~N}=18)$ and EDSS $>2(\mathrm{~N}=11)$ corresponding to feature notation _ 1 , and _2 respectively (see also TABLE I). Interquartile range (IQR) values are shown above the box plots. In each plot we display the median, lower, and upper quartiles and confidence interval around the median. Straight lines connect the nearest observations within 1.5 of the IQR of the lower and upper quartiles.

\subsection{Manual Delineations and Visual Perception}

All MRI-detectable brain lesions were identified and segmented by an experienced MS neurologist and confirmed by a radiologist. Only well-defined areas of hyperintensity on T2-weighted MR images were considered as MS lesions. The neurologist manually delineated (using the mouse) the brain lesions by selecting consecutive points at the visually defined borders between the lesions and the adjacent NAWM on the acquired transverse T2-weigted sections. Similar regions corresponding to NAWM were delineated contralaterally to the detected MS lesions. The manual delineations were performed using a graphical user interface implemented in MATLAB developed by our group. Manual segmentation by the MS expert was performed in a blinded manner, without the possibility of identifying the subject or the clinical findings. The selected points and delineations were saved to be used for texture analysis. 


\subsection{Texture Analysis}

In this study texture features and shape parameters were extracted from all MS lesions detected and segmented [8], [9], while texture features were also calculated for the NAWM ROIs. The overall texture and shape features for each subject were then estimated by averaging the corresponding values for all lesions for each subject. The following texture features were extracted: (i) Statistical Features: a) mean, b) variance, c) median value, d) skewness, e) kurtosis, f) energy and g) entropy. (ii) Spatial Gray Level Dependence Matrices (SGLDM) as proposed by Haralick et al. [17]: a) angular second moment (ASM), b) contrast, c) correlation, d) sum of squares variance (SOSV), e) inverse difference moment (IDM), f) sum average (SA), g) sum variance (SV), h) sum entropy (SE), i) entropy, j) difference variance (DV), k) difference entropy (DE), and 1) information measures of correlation (IMC). For a chosen distance $\mathrm{d}$ (in this work $\mathrm{d}=1$ was used) and for angles $\theta=0^{\circ}, 45^{\circ}, 90^{\circ}$, and $135^{0}$, we computed four values for each of the above texture measures. Each feature was computed using a distance of one pixel. Then for each feature the mean values and the range of values were computed, and were used as two different features sets. (iii) Gray Level Difference Statistics (GLDS) [18]: a) homogeneity, b) contrast, c) energy, d) entropy, and e) mean. The above features were calculated for displacements $\delta=(0,1),(1,1),(1,0),(1,-1)$, where $\delta \equiv(\Delta x, \Delta y)$, and their mean values were taken. (iv) Neighbourhood Gray Tone Difference Matrix (NGTDM) [19]: a) coarseness, b) contrast, c) busyness, d) complexity, and e) strength. (v) Statistical Feature Matrix (SFM) 20: a) coarseness, b) contrast, c) periodicity, and d) roughness. (vi) Laws Texture Energy Measures (LTEM) [18]: LL-texture energy from LL kernel, EE-texture energy from EE-kernel, SS-texture energy from SS-kernel, LE-average texture energy from LE and EL kernels, ES-average texture energy from ES and SE kernels, and LS-average texture energy from LS and SL kernels. (vii) Fractal Dimension Texture Analysis (FDTA) [21]: The Hurst coefficients for dimensions 4, 3 and 2 were computed. (viii) Fourier Power Spectrum (FPS) [18], [20]: a) radial sum, and b) angular sum. (vii) Shape Parameters: a) $\mathrm{X}$-coordinate maximum length, b) $\mathrm{Y}$-coordinate maximum length, c) area, d) perimeter, e) perimeter2/area, f) eccentricity, g) equivalence diameter, h) major axis length, i) minor axis length, $\mathrm{j}$ ) centroid, k) convex area, and l) orientation.

\subsection{Models Support Vector Machines Classification}

Classification analysis was carried out to classify brain MS lesions delineated on the baseline MRI scans into two classes according to the EDSS score that each patient was allocated two years following initial diagnosis: (i) MS subjects with $\mathrm{EDSS} \leq 2$, and (ii) MS subjects with an EDSS $>2$. Thus, the classification goal was to differentiate between lesions that were subsequently associated with mild (EDSS $\leq 2)$ or advanced disability (EDSS $>2$ ). The classification was applied on 30 subjects (15 males and 15 females).

Each classifier was implemented in Matlab, using Support Vector Machines (SVM). The SVM network was investigated using Gaussian Radial Basis Function 
kernels [22]; this was decided as the rest of the kernel functions could not achieve satisfactory results. The Parameters for the classifier were selected using ten-fold cross validation for each set. Parameter C of SVM was 1 . The leave-one-out estimate was used for validating the classification models.

The performance of the classifier models were measured using the receiver operating characteristics (ROC) curve parameters [23]. The parameters calculated for ROC curves are: (i) true positives (TP) when the system correctly classifies subjects as $\mathrm{EDSS} \leq 2$, and EDSS $>2$, (ii) false positives (FP) where the system wrongly classifies subjects as EDSS $>2$ while they are in the group of EDSS $\leq 2$, (iii) true negatives (TN) when the system correctly classifies subjects as EDSS $\leq 2$. We also compute the Sensitivity (SE) which is the likelihood that a subject with EDSS $>2$ will be detected given that it is EDSS $>2$ and Specificity (SP) which is the likelihood that a subject will be classified as EDSS $\leq 2$ given that he/she is EDSS $\leq 2$. For the overall performance, we provide the correct classification (CC) rate which gives the percentage of correctly classified subjects.

\section{Results}

We present in Fig. 2 box plots of the lesion texture features mean (Mean0_1, Mean0_2) median (Median0_1, Median0_2) and sum average (SA0_1, SA0_2) for 0 months for $\mathrm{EDSS} \leq 2\left(\_1\right)(\mathrm{N}=18)$ and $\mathrm{EDSS}>2\left(\_2\right)(\mathrm{N}=11)$.

Table 1. Statistical Analysis Of The Texture Features For Lesions And NAWM Subjects With EDSS $\leq 2(\mathrm{~N}=18)$ and EDSS $>2(\mathrm{~N}=11)$ Based on The Mann Whitney (first row) and the Wilcoxon (2nd row) Rank Sum Tests at $\mathrm{p}<0.05$. Significant Difference is Depicted With S And Non Significant Difference is Depicted With NS. The p Values Are Shown in Parentheses.

\begin{tabular}{c|c|c}
\hline Feature & Lesions & NAWM \\
\hline Mean & S (0.03) & NS $(0.18)$ \\
Median & S (0.03) & NS $(0.17)$ \\
STD & NS $(0.83)$ & NS $(0.07)$ \\
Contr & NS $(0.16)$ & NS $(0.36)$ \\
SOSV & NS $(0.83)$ & NS $(0.55)$ \\
Entr & NS (0.06) & NS $(0.58)$ \\
IDM & S (0.01) & NS $(0.36)$ \\
SA & S (0.04) & NS $(0.17)$ \\
SV & NS (0.83) & NS $(0.19)$ \\
DV & NS (0.44) & NS $(0.18)$ \\
DE & NS (0.35) & NS $(0.77)$ \\
\hline
\end{tabular}

NAWM: Normal appearing white matter, STD: Standard deviation, Contr.: Contrast, SOSV: Sum of square variance, Entr: Entropy, IDM: Inverse difference moment, SA: Sum average, SV: Sum variance, DE: Difference entropy. 
Table 2. Classification Results Using The Texture And Shape Feature Set And The Support Vector Machines Classifier For Two Classes: EDSS $\leq 2$ and EDSS $>2(\mathrm{~N}=30)$. The Results Are Presented Using the ROC Measures: Percentage of correct classifications (\%CC), percentage of false positives $(\% \mathrm{FP})$, percentage of false negatives $(\% \mathrm{FN})$, percentage sensitivity $(\% \mathrm{SE})$ and percentage specificity $(\% \mathrm{SP})$.

\begin{tabular}{l|c|c|c|c|c}
\hline Feature Group & \% CC & \%FP & \%FN & \%SE & \%SP \\
\hline FOS & 43 & 47 & 67 & 33 & 53 \\
\hline SGLDM(mean) & 63 & 53 & 20 & 80 & 47 \\
\hline SGLDM(range) & 63 & 53 & 20 & 80 & 47 \\
\hline GLDS & 67 & 47 & 20 & 80 & 53 \\
\hline NGTDM & 67 & 40 & 27 & 73 & 60 \\
\hline SFM & 67 & 40 & 27 & 73 & 60 \\
\hline TEM & 67 & 40 & 27 & 73 & 60 \\
\hline FDTA & 60 & 33 & 7 & 53 & 67 \\
\hline FPS & 60 & 53 & 27 & 73 & 47 \\
\hline NGTDM \& SFM & 73 & 27 & 27 & 73 & 73 \\
\hline GLDS \& NGTDM \& SFM & 77 & 27 & 20 & 80 & 73 \\
\hline SHAPE & 53 & 53 & 40 & 60 & 47 \\
\hline
\end{tabular}

Statistical analysis was carried out for all texture features given in section 2.3 for lesions and NAWM for EDSS $\leq 2$ versus EDSS $>2$. The results of selected features, including those that showed significant difference are tabulated in Table 1. For lesions, features mean, median, IDM and SA could differentiate between EDSS $\leq 2$ and EDSS $>2$ (see also Fig. 2). For the NAWM it is shown that there are no significant differences between subjects at 0 months with $\mathrm{EDSS}>2$ and $\mathrm{EDSS} \leq 2$, for all texture features.

Table 2 presents the lesion classification results. According to Table II the best correct classification rate was achieved as a combination of GLDS, NGTDM and SFM feature sets and was $77 \%$.

\section{Discussion}

The objective of our study was to investigate texture classification analysis in an effort to differentiate between MS brain lesions that, in a subsequent stage, will be associated with advanced diseased disability from those lesions that will be related to a mild disability score.

Table 1 showed that there are some texture features that can be possibly used to differentiate between subjects with mild (EDSS $\leq 2)$ and advanced (EDSS $>2$ ) MS disease states. These features are mean, median, IDM and SA. Table 2 showed that the combination of GLDS, NGTDM \& SFM texture features gave a \% of CC rate of $77 \%$ when classifying subjects according to their long-term disability status.

These results can be compared with another study carried out by our group [14], where AM-FM feature analysis was used but on a slightly larger set of subjects. The findings suggest that AM-FM characteristics succeed in differentiating (i) between 
NWM and lesions, (ii) between NAWM and lesions, and (iii) between NWM and NAWM. A support vector machine (SVM) classifier succeeded in differentiating between patients that, two years after the initial MRI scan, acquired an EDSS $\leq 2$ from those with EDSS $>2$ (correct classification rate $=86 \%$ ). The best classification results were obtained from including the combination of the low-scale instantaneous amplitude (IA) and instantaneous frequency (IF) magnitude with the medium-scale IA. Among all AM-FM features, the medium frequency IA histogram alone gave the best AUC results (AUC=0.76). Also the best \% of CC achieved was $86 \%$. The IA histograms from all frequency scales contributed to a multi-classifier system that gave $86 \%$ correct classification rate. The best classifier results also used the IF magnitude from both the low and the high frequency scales.

To the best of our knowledge, no other studies were carried out for differentiating between the aforementioned two disability scores. Several studies were carried out for differentiating and classifying NWM, and or NAWM, and lesions, as these are summarized below.

Various studies have been performed in order to establish a relationship between the various gray levels and texture features [4]-[11]. In [5] MRI texture analysis based on statistical, autoregressive model, and wavelet-derived texture analysis was performed on 30 MS subjects. The classification accuracy between MS lesions, NAWM and plaques NWM, was 96\%-100\%.

Likewise in [24], texture analysis was performed on MR images of MS subjects and normal controls and a combined set of texture features were explored in order to better discriminate tissues between MS lesions, NAWM and NWM. The results demonstrated that with the combined set of texture features, classification was perfect $(100 \%)$ between MS lesions and NAWM (or NWM), less successful (88.89\%) among the three tissue types and worst (58.33\%) between NAWM and NWM. Furthermore, it was shown that compared with GLCM-based features, the combined set of texture features were better at discriminating MS lesions and NWM, equally good at discriminating MS lesions and NAWM and at all three tissue types, but less effective in classification between NAWM and NWM. The study suggested that texture analysis with the combined set of texture features may be equally good or more advantageous than the commonly used GLCM-based features alone in discriminating MS lesions and NWM/NAWM and in supporting early diagnosis of MS.

$\mathrm{Yu}$ et al. [11] performed textural feature analysis to discriminate active and nonactive MS lesions in a study of 8 subjects with relapsing remitting MS, by using linear discriminant analysis using 42 first- and second order statistical textural features. Applications of the run-length method have been very limited compared with other methods, yet $\mathrm{Yu}$ et al. found that run-length matrix features actually outperformed gray-level co-occurrence matrix features in the identification of active MS lesions, with run-length matrix features distinguishing active from inactive lesions with $88 \%$ sensitivity and $96 \%$ specificity. Conversely, none of the gray-level co-occurrence features provided any discrimination between lesion subtypes.

In another study [12], it was shown that texture analysis can achieve high classification accuracy $(>=90 \%)$ in tissue discrimination between MS lesions and NAWM.

In [25] the authors tried to objectively identify possible differences in the signal characteristics of benign and malignant soft tissue masses on MRI by means of 
texture analysis and to determine the value of these differences for computer-assisted lesion classification. Fifty-eight subjects with histologically proven soft tissue masses (benign, $\mathrm{n}=30$; malignant, $\mathrm{n}=28$ ) were included and their masses were texture analyzed. The best results of soft tissue masses classification were achieved using texture information from short tau inversion recovery images, with an accuracy of $75.0 \%$ (sensitivity, 71.4\%; specificity, 78.3\%) for the k-NN classifier, and an accuracy of $90.5 \%$ (sensitivity, 91.1\%; specificity, 90.0\%) for the artificial neural network classifier. The authors concluded that, texture analysis revealed only small differences in the signal characteristics of benign and malignant soft tissue masses on routine MRI.

\section{Future Work}

Further research work on a larger number of subjects is required for validating the results of this study and for finding additional shape and texture features that may provide information for longitudinal monitoring of the lesions on the initial MRI scan of the brain of patients with CIS; and improve the final classification rate. Additionally the use of other classifiers could suggest a better classification a scheme and will be examined. Finally the proposed methodology could be possibly used for the assessment of subjects at risk of developing future neurological events and disease progression as measured by increased EDSS score.

Acknowledgment. This work was supported through the project Quantitative and Qualitative Analysis of MRI Brain Images TПE/OPIZO/0308(BIE)/15, 12/200812/2010, of the Program for Research and Technological Development 2007-2013, co funded by the Research Promotion Foundation of Cyprus.

\section{References}

1. Fazekas, F., Barkof, F., Filippi, M., Grossman, R.I., Li, D.K.B., McDonald, W.I., McFarland, H.F., Patty, D.W., Simon, J.H., Wolinsky, J.S., Miller, D.H.: The contribution of magnetic resonance imaging to the diagnosis of multiple sclerosis. Neur. 53, 44-456 (1999)

2. McDonald, W.I., Compston, A., Edan, G., et al.: Recommended diagnostic criteria for multiple sclerosis: guidelines from the international panel on the diagnosis of multiple sclerosis. Ann. Neurol. 50, 121-127 (2001)

3. Bakshi, R., Thompson, A.J., Rocca, M.A., et al.: MRI in multiple sclerosis: current status and future prospects. Lancet Neurol. 7, 615-625 (2008)

4. Kassner, A., Thornhill, R.E.: Texture analysis: A review of neurologic MR imaging applications. Am. J. Neuroradiol. 31, 809-816 (2010)

5. Harrison, L.C.V., Raunio, M., Holli, K.K., Luukkaala, T., Savio, S., et al.: MRI Texture analysis in multiple sclerosis: Toward a clinical analysis protocol. Acad. Radiol. 17, 696707 (2010)

6. Herlidou-Meme, S., Constans, J.M., Carsin, B., Olivie, D., Eliat, P.A., et al.: MRI texture analysis on texture test objects, normal brain and intracranial tumors. Mag. Res. Imag. 21, 989-993 (2003) 
7. Mathias, J.M., Tofts, P.S., Losseff, N.A.: Texture analysis of spinal cord pathology in multiple sclerosis. Magn., Reson. Med. 42, 929-935 (1999)

8. Loizou, C.P., Pattichis, C.S., Seimenis, I., Eracleous, E., Schizas, C.N., Pantziaris, M.: Quantitative analysis of brain white matter lesions in multiple sclerosis subjects: Preliminary findings. In: IEEE Proc., 5th Int. Conf. Inf. Techn. Appl. Biomed., ITAB, Shenzhen, China, May 30-31, pp. 58-61 (2008)

9. Loizou, C.P., Pattichis, C.S., Seimenis, I., Pantziaris, M.: Quantitative analysis of brain white matter lesions in multiple sclerosis subjects. In: 9th Int. Conf. Inform. Techn. Applic. Biomed., ITAB, Larnaca, Cyprus, November 5-7, pp. 1-4 (2009)

10. Collewet, G., Strzelecki, M., Marriette, F.: Influence of MRI acquisition protocols and image intensity normalization methods on texture classification. Magn. Reson. Imag. 22, 81-91 (2004)

11. Yu, O., Mauss, Y., Zollner, G., Namer, I.J., Chambron, J.: Distinct patterns of active and non-active plaques using texture analysis of brain NMR images in multiple sclerosis patients: Preliminary results. Magn. Reson. Imag. 17(9), 1261-1267 (1999)

12. Zhang, J., Wang, L., Tong, L.: Feature reduction and texture classification in MRI-Texture analysis of multiple sclerosis. In: IEEE/ICME Conf. Complex Med. Eng., pp. 752-757 (2007)

13. Meier, D.S., Guttman, C.R.G.: Time-series analysis of MRI intensity patterns in multiple sclerosis. NeuroImage 20, 1193-1209 (2003)

14. Loizou, C.P., Murray, V., Pattichis, M.S., Seimenis, I., Pantziaris, M., Pattichis, C.S.: Multi-scale Amplitude Modulation-Frequency Modulation (AM-FM) texture analysis of multiple sclerosis in brain MRI images. IEEE Trans. Inform. Tech. Biomed. 15(1), 119129 (2011)

15. Thompson, A.J., Hobart, J.C.: Multiple sclerosis: assessment of disability and disability scales. J. Neur. 254(4), 189-196 (1998)

16. Loizou, C.P., Pantziaris, M., Seimenis, I., Pattichis, C.S.: MRI intensity normalization in brain multiple sclerosis subjects. In: ITAB 2009, 9th Int. Conf. on Inform. Techn. And Applic. in Biomed., Larnaca, Cyprus, November 5-7, pp. 1-5 (2009)

17. Haralick, R.M., Shanmugam, K., Dinstein, I.: Texture features for image classification. IEEE Trans. Syst., Man., and Cybernetics SMC-3, 610-621 (1973)

18. Weszka, J.S., Dyer, C.R., Rosenfield, A.: A comparative study of texture measures for terrain classification. IEEE Trans. Syst., Man. Cybern. SMC-6, 269-285 (1976)

19. Amadasun, M., King, R.: Textural features corresponding to textural properties. IEEE Trans. Syst. 19(5), 1264-1274 (1989)

20. Wu, C.M., Chen, Y.C., Hsieh, K.-S.: Texture features for classification of ultrasonic images. IEEE Trans. Med. Imag. 11, 141-152 (1992)

21. Mandelbrot, B.B.: The Fractal Geometry of Nature. Freeman, San Francisco (1982)

22. Cristianini, N., Shawe-Taylor, J.: An Introduction to Support Vector Machines and Other Kernel-based Learning Methods, 1st edn. Cambridge University Press, Cambridge (2000)

23. Ebrchart, R.C., Dobbins, R.W.: Neural Networks PC Tools A Practical Guide. Academic Pr., New York (1990)

24. Zhang, J., Tong, L., Wang, L., Lib, N.: Texture analysis of multiple sclerosis: a comparative study. Magn. Res. Imag. 26(8), 1160-1166 (2008)

25. Mayerhoefer, M.E., Breitenseher, M., Amannd, G., Dominkuse, M.: Are signal intensity and homogeneity useful parameters for distinguishing between benign and malignant soft tissue masses on MR images? Objective evaluation by means of texture analysis. Magn. Res. Imag. 26, 1316-1322 (2008) 